\title{
Économie d'eau des toilettes, une approche crédible de réduction du déficit en eau potable de la ville d'Abidjan (Côte d'Ivoire)
}

\author{
C. TA BI BOTI ${ }^{1 *}$, S. KENFACK ${ }^{2}$, T. GNAGNE ${ }^{1,3}$ et G. SORO ${ }^{1}$ \\ ${ }^{1}$ Laboratoire de Géosciences et Environnement, Unité de Formation et de Recherche des Sciences et \\ Gestion de l'Environnement (UFR-SGE), Université Nangui Abrogoua, 02 BP 801, Abidjan 02, Côte d'Ivoire. \\ ${ }^{2}$ Association Africaine de l'Eau (AAE), Abidjan, Côte d'Ivoire. \\ ${ }^{3}$ Eau et Assainissement pour l'Afrique (EAA), Ouagadougou, Burkina-Faso. \\ "Auteur correspondant; E-mail: c.boti14@gmail.com; Tel: +22548968541
}

\section{RESUME}

La population abidjanaise s'est fortement accrue ces dernières années. Cette croissance effrénée a entraîné un déficit d'eau dû à la forte demande. Afin de palier à cette pénurie d'eau, il est proposé l'alternative d'économie d'eau à domicile à travers l'utilisation de modèles de chasses d'eau économiques dans les ménages. L'évaluation de cette alternative s'est faite à travers une enquête sur un échantillon de 100 ménages puis le suivi de la consommation avant et après introduction des chasses d'eau économique auprès de 5 ménages. Les résultats révèlent que la consommation spécifique journalière en eau potable dans la ville d'Abidjan est estimée à 97 litres sans les chasses d'eau économique tandis qu'avec ce dispositif d'économie d'eau, la consommation chute de 97 litres à 86 litres au moins et au plus 70 litres par personne et par jour. En extrapolation sur la population totale, cela permettrait de réaliser une économie d'eau journalière globale allant de $37000 \mathrm{~m}^{3}$ à $94000 \mathrm{~m}^{3}$ d'eau dans l'ensemble de la ville d'Abidjan.

(C) 2019 International Formulae Group. All rights reserved

Mots clés: Ressource en eau, pénurie d'eau, chasse d'eau économique, consommation d'eau du ménage.

\section{Saving water for sanitary use, a credible approach to reducing the deficit in urban drinking water of Abidjan (Côte d'Ivoire)}

\begin{abstract}
Abidjan's population has greatly increased in recent years. This unbridled growth has led to a lack of water due to strong demand. To overcome this shortage of water, it is proposed the alternative of saving water at home through the use of models of flushing economic water in households. The evaluation of this alternative was done through a survey of a sample of 100 households and the monitoring of consumption before and after the introduction of economic flushing with 5 households. The results reveal that the daily specific consumption of drinking water in the city of Abidjan is estimated at 97 litters without economical flushing, while with this watersaving device consumption drops from 97 litters to 86 litters at least 70 litters per person per day. Extrapolating to
\end{abstract}


the total population, this would lead to an overall daily water saving of $37,000 \mathrm{~m}^{3}$ to $94,000 \mathrm{~m}^{3}$ of water throughout of Abidjan.

(C) 2019 International Formulae Group. All rights reserved

Keywords: Water resource, water scarcity, economic flush, household water consumption.

\section{INTRODUCTION}

L'accès à l'eau potable en quantité satisfaisante demeure incontestablement un élément de survie majeur qui conditionne le développement économique et social des collectivités humaines.

En Côte d'Ivoire, le taux de couverture des besoins en eau potable en 2014 était évalué à $60 \%$ en milieu urbain. En milieu rural, il était à $13 \%$ en hydraulique villageoise amélioré (HVA) et à $76 \%$ en hydraulique villageoise (PND 2016-2020).

Dans le cas de la ville d'Abidjan qui abrite aujourd'hui 4,4 millions d'habitants, soit $20 \%$ de la population ivoirienne (INS, 2014 ) et $39 \%$ de la population urbaine, la demande en eau ne cesse de croître au fil des années, tandis que la disponibilité en quantité et en qualité de la nappe d'Abidjan, qui sert de ressources en eaux depuis plus de 50 ans se réduit progressivement tant en raison des aléas climatiques, de la pollution, que de l'accroissement effréné de la population. En effet, du fait de la forte croissance démographique (croissance de 2,6\% entre 1998 à 2014), la population abidjanaise est passée de 2,9 millions en 1998 à 4,4 millions en 2014 (INS, 2014). La demande journalière s'est ainsi accrue, passant de $180000 \mathrm{~m}^{3}$ en 1983 pour une production de $260000 \mathrm{~m}^{3}$ à $461000 \mathrm{~m}^{3}$ contre une production de 311000 (Ettien, 2010), soit une production supplémentaire de $131000 \mathrm{~m}^{3} /$ jour en 27 ans contre une demande de $281000 \mathrm{~m}^{3} /$ jour. Cette situation a engendré une pression sans précédent sur l'industrie de l'eau entrainnant un déficit journalier de production d'environ $150000 \mathrm{~m}^{3}$. Ce déficit représente le besoin en eau potable d'une population estimée à 1,5 millions d'habitants qui n'ont pas accès à l'eau potable dans la capitale ivoirienne, si l'on considère une demande de 100 litres par jour et par personne.
Face à cette situation, la première option qui a été celle appliquée par le gouvernement est de réaliser des investissements pour faire venir l'eau de la ville de Bonoua située à $59 \mathrm{~km}$ d'Abidjan. Un investissement de 90 milliards de francs CFA pour une durée d'exploitation maximale de 15 ans. Cette approche revient à se tourner après le terme des 15 ans vers l'exploitation d'autres ressources en eaux.

Une deuxième alternative peut porter sur la réalisation d'économie d'eau qui a l'avantage d'exercer moins de pression sur la ressource en eau et qui peut d'ailleurs être la moins coûteuse. C'est dans cette optique que la présente étude s'est fixé comme objectif d'évaluer l'économie d'eau potable réalisable dans la ville d'Abidjan par l'utilisation des chasses d'eau sanitaires économique dans les ménages.

\section{MATÉRIEL ET MÉHODES Zone d'étude}

L'étude a été faite dans la ville d'Abidjan. Abidjan est la capitale économique de la Côte d'Ivoire. Elle est située au sud-est du pays dans la région des lagunes, entre les latitudes Nord $5^{\circ} 12^{\prime}$ et $5^{\circ} 30^{\prime}$ et les longitudes Ouest $3^{\circ} 48^{\prime}$ et $4^{\circ} 12^{\prime}$ (Figure 1). Depuis 1978, elle comprend dix communes (SORO et al., 2010) et sa population est estimée en 2014 à 4,4 millions d'habitants (INS, 2014).

Les formations géologiques du soussol abidjanais appartiennent au bassin sédimentaire côtier. Le bassin sédimentaire est d'âge crétacé supérieur à quaternaire (BNETD, 2015).

Du point de vue hydrogéologique, le sous-sol abidjanais est composé d'aquifères homogènes et très perméables. Ces couches révèlent trois types de nappes à savoir l'aquifère du Quaternaire qui contient la 
nappe la plus vulnérable à la pollution avec un niveau piézométrique très proche de la surface du sol, l'aquifère du Continental Terminal d'âge mio-pliocène ou Nappe d'Abidjan, d'où est alimenté l'ensemble de la ville d'Abidjan en eau potable et l'aquifère du Maestrichtien avec une nappe situé à 200 $\mathrm{m}$ de profondeur dans les bancs calcaires du Maestrichtien (BNETD, 2015).

\section{Matériel}

\section{Les chasses d'eau peu économiques en eau}

Les chasses d'eau rencontrées dans les ménages sont des chasses d'eau à bouton poussoir (Figure 2a) ou à tirette (Figure 2b) avec la possibilité d'exercer une seule action sur la chasse et qui libère presque toute l'eau du réservoir.

\section{Les chasses d'eau économiques}

Des chasses d'eau sanitaires économiques (Figure 2c) ont été installées dans certains des ménages enquêtés. Ces chasses d'eau économiques sont des chasses d'eau à bouton poussoir avec la possibilité d'exercer deux actions sur la chasse. Une première action consistant à appuyer sur le bouton et le relâcher aussitôt (après 1 à 2 secondes environ). Cette première action permet l'évacuation des besoins autres que les selles et les vomissements. La seconde action permet d'évacuer les selles et vomissement, elle consiste à appuyer sur le bouton et le maintenir jusqu'à l'évacuation 3 à 5 secondes environ (Figure 3 ).

\section{Les compteurs d'eau}

Des compteurs d'eau utilisés pour les relevés les consommations en eau des ménages. Des précisions ont été vérifiées en effectuant trois séries de tests avec deux récipients de capacités connues d'avance (10 et 20 litres). Chaque récipient était rempli trois fois de suite au robinet et le volume d'eau recueilli vérifié par la différence des relevés d'index du compteur.

\section{Méthodologie}

\section{Première phase : Enquête ménages}

La première phase a duré 45 jours (du 20 novembre 2015 au 04 janvier 2016). Elle a consisté à recueillir des données auprès des ménages à travers une enquête. Elle a permis de connaître les caractéristiques des ménages et des habitats, le niveau des équipements des ménages et leur impact sur la consommation en eau, la consommation en eau des différents usages domestiques ainsi que la consommation d'eau journalière par habitant à partir de ces usages.

Pour ce faire, un questionnaire d'enquête composé de trois parties a été élaboré. La première partie a porté sur les caractéristiques du ménage. La deuxième partie a permis d'obtenir des informations sur l'état du réseau d'eau et l'appréciation du ménage sur la facturation de sa consommation. La dernière partie a permis de quantifier la consommation d'eau : cuisine, bain, WC, nettoyage et autres. La quantification des consommations d'eau de ces différents usages s'est faite d'une part à travers les récipients (seaux, bassines, etc...) couramment utilisés dans les ménages dont les capacités sont connues d'avance. Ces différents récipients ont permis de quantifier particulièrement l'eau des usages tels que la cuisine, la boisson, le nettoyage, la lessive et la vaisselle. D'autres part, les consommations en eau des usages WC et douche ont été quantifié à travers des séries de deux à trois tests de relevés d'indexe de compteurs d'eau des ménages disposant de $\mathrm{WC}$ a chasse d'eau et de tête de conne de douche puis à travers des seaux de 6, 10, 15 et 20 litres pour les ménages disposant de WC sans chasse d'eau et n'ayant pas de tête de colonne de douche.

Afin que l'enquête se déroule dans de bonnes conditions, et pour une instruction informée, une «lettre de consentement» a été remise à chaque ménage avant l'administration du questionnaire, et souvent quelques jours avant notre visite.

Par ailleurs, pour que les données d'enquêtes soient crédibles, un échantillon représentatif a été constitué par la méthode d'échantillonnage non exhaustive aléatoire simple stratifiée. La taille de l'échantillon a été déterminée à partir de la formule cidessous : 


$$
n=\frac{(1,96)^{2} * N}{(1,96)^{2}+\left(l^{2} *(N-1)\right)}
$$

Où $\boldsymbol{n}=$ taille de l'échantillon; $\mathbf{N}=$

taille de la population observée (Population d'Abidjan);

$l=$ fourchette exprimant la marge d'erreur et 1,96 = écart-type correspondant à un seuil de confiance de $95 \%$.

La marge d'erreur retenue pour cette étude a été de $10 \%$, ce qui correspond à une taille d'échantillon $\mathrm{n}=96$ ménages. $\mathrm{Ce}$ nombre a été par la suite arrondi à 100 .

Les

caractéristiques sociodémographiques, les habitats et les installations sanitaires observées dans les ménages pendant l'enquête ont permis de structurer l'échantillon en trois principales strates: les ménages de bas standing, les ménages de moyens standing et les ménages de haut standing. L'enquête s'est déroulée dans six des dix communes que compte la ville d'Abidjan. Il s'agit d'une part des communes d'Adjamé, Attécoubé et Treichville abritant principalement les ménages de bas et de moyen standing et d'autre part des communes de Cocody, Marcory où l'on trouve le plus souvent les ménages de haut et moyen standing puis la commune de Yopougon qui abrite les trois niveaux de standing.

- Les ménages de bas standing sont rencontrés dans les quartiers précaires avec voiries mal tracées, sans de véritables réseaux d'assainissements. Les eaux usées domestiques sont déversées à l'aire libre ou dans les puisards, les défécations se font souvent à l'air libre ou sont collectées dans des fosses septiques mal entretenues. Les habitats sont faits en dur, en terre, ou en bois et sans délimitation (clôtures).

- Les ménages de moyen standing sont rencontrés dans les quartiers disposant d'une voirie bien tracée et de réseaux d'assainissements, mais souvent mal entretenus. Les habitats sont généralement de deux ou trois pièces (une ou deux chambres- salon), construites en dur et souvent délimités par des clôtures.

- Les ménages de haut standing sont rencontrés dans les quartiers résidentiels, les voies sont bien tracées et bitumées pour la plupart. Les eaux fluviales sont collectées et drainées par de véritables réseaux d'assainissements bien dimensionnées et entretenus. Les eaux usées domestiques et les selles sont collectées dans les fosses septiques bien entretenues. Les habitats sont de type villa ou duplex bien délimités par des clôtures.

La répartition des échantillons de ménages à enquêter par commune s'est faite proportionnellement au nombre de ménages existant dans chacune des six communes selon la formule :

$P=\left(\frac{\text { nombre de ménage de la commune }}{\text { nombre total de ménage de l'enssemble des communes }}\right) * n$

Avec $\boldsymbol{P}=$ Proportion de ménage à enquêter dans la commune

$$
\boldsymbol{n}=\text { Taille totale de l'échantillon }
$$

Seconde phase: sélection des ménages, suivi des consommations et installation des chasses économique

La seconde phase a duré 66 jours (du 9 janvier au 28 mars 2016) et a consisté tout d'abord à identifier parmi les 100 ménages enquêtés ceux disposant de WC à chasses d'eau ordinaires fonctionnelles et ne présentant pas de fuite d'eau dans le réseau domestique puis d'en sélectionner 5 disponibles à poursuivre d'étude (cette étape a duré 10 jours). Dans ces ménages sélectionnés, le suivi des consommations d'eau par relevés d'indexe de compteur d'eau s'est effectué pendant 52 jours, soit 21 jours avant (lorsque le ménage utilisait ses équipements sanitaires habituels), 14 jours pour les installations des mécanismes économiques suivi d'apprentissage des ménages à une meilleure utilisation des nouveaux mécanismes et 21 autres jours de suivi de la consommation après installations. 


\section{Analyse des données}

Les données issues du questionnaire ont été dépouillées avec le logiciel EpiData version 3.1. Une analyse descriptive simple des variables statistiques ainsi que le croisement deux à deux de certaines variables ont été effectué sous IBM SPSS statistica version 20. Les traitements ont permis de générer des tableaux relatifs aux différents critères d'intérêt en vue d'une meilleure interprétation. Les données de consommation d'eau potable relevées sur les compteurs d'eau ont été saisies, organisées et analysées à l'aide du logiciel Microsoft Excel 2013 et représentées sous formes de tableaux.

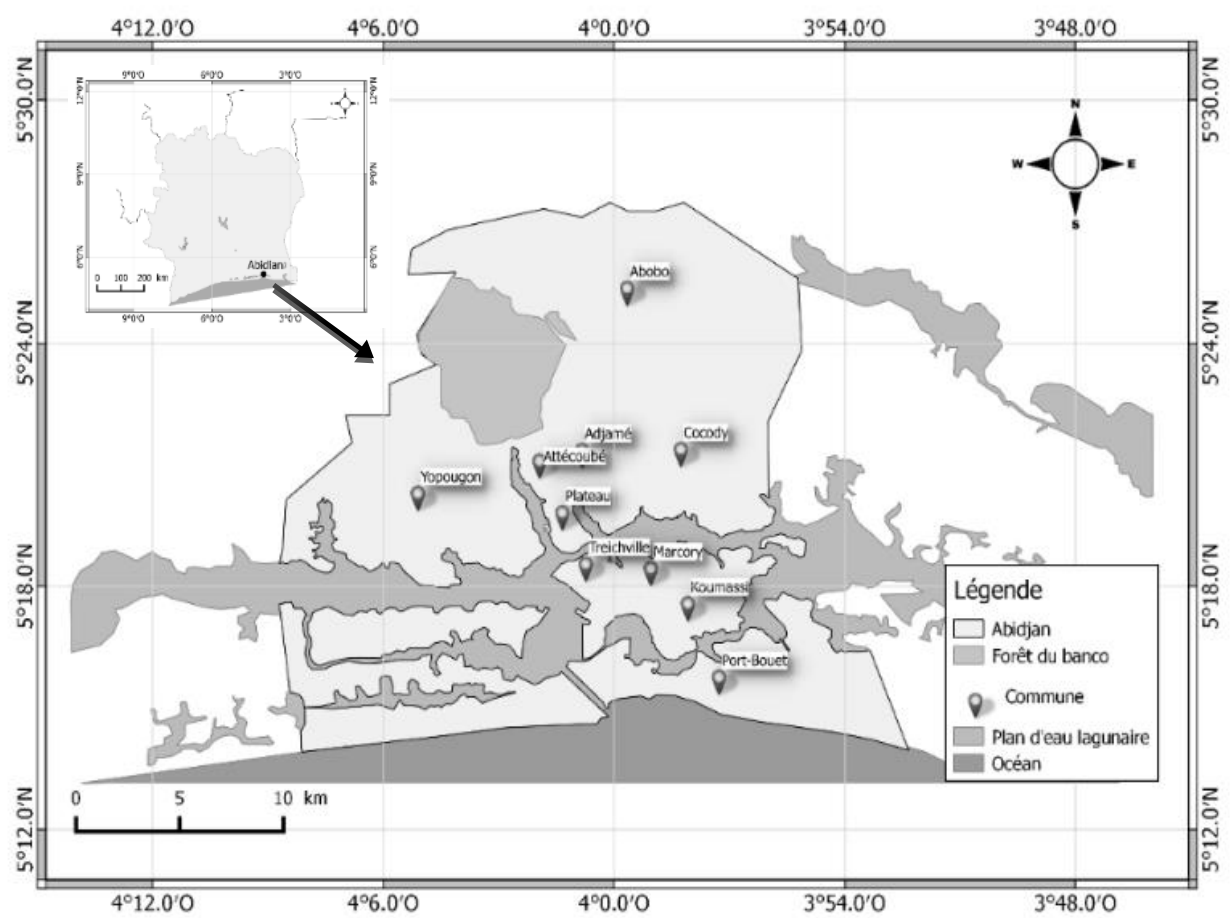

Figure 1 : Localisation géographique de la zone d'étude.
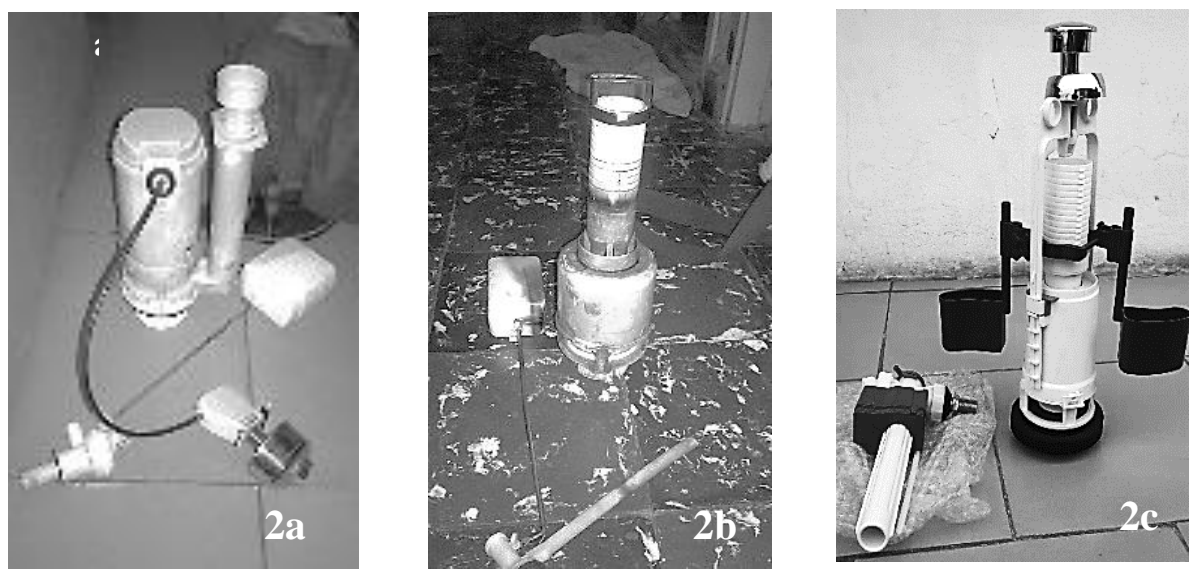

Figure 2 : Différents mécanismes de chasses d'eau utilisés. 


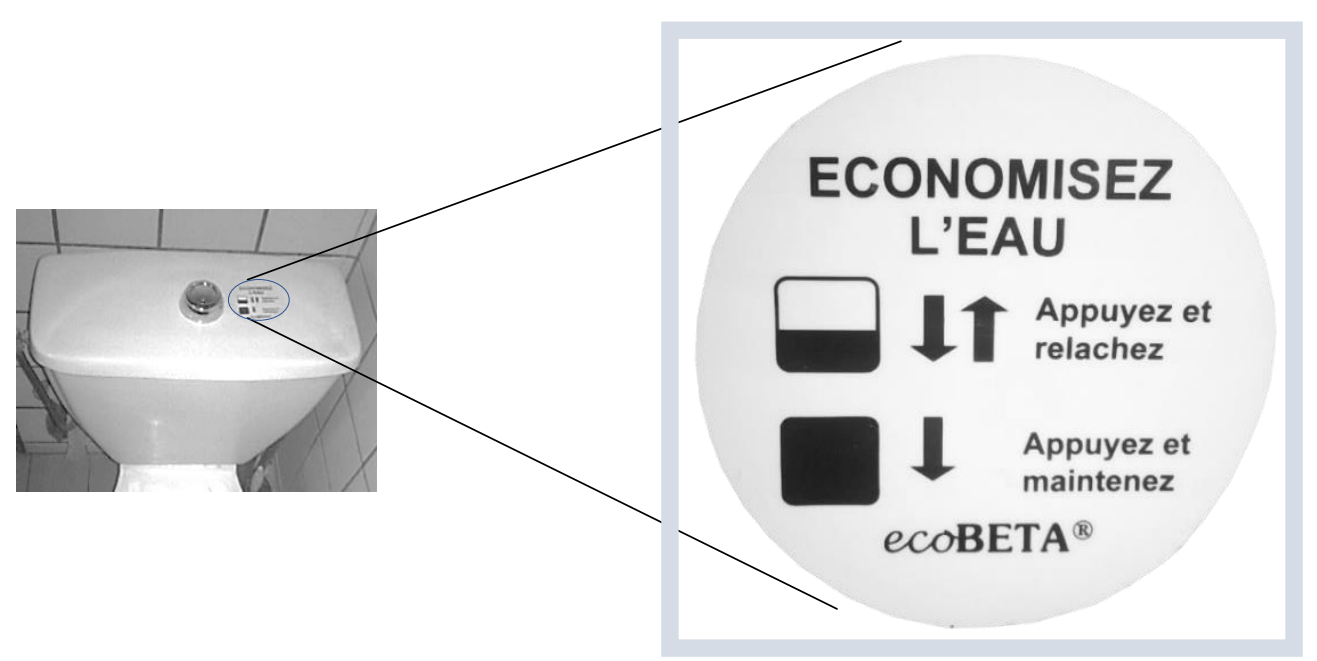

Figure 3 : Autocollant indiquant le mode d'utilisation de la chasse d'eau économique.

\section{RESULTATS}

\section{Consommation domestique de l'eau}

$\mathrm{La}$ moyenne de consommation journalière en eau par personne et pour l'ensemble des ménages est de 97 litres. Cette valeur moyenne varie selon le standing des différents ménages. Elle est de 132 litres par personne pour les hauts standings et de 88 litres par personne pour les bas standings (Tableau 1).

Par ailleurs, en ce qui concerne les consommations en eau potable pour les différents besoins domestiques exprimées en litres, on constate qu'il n'existe pas une très grande différence entre les consommations moyennes et les consommations médianes (Tableau 2). Cela signifie que la consommation en eau potable selon le niveau de standing pour chaque usages domestiques est la même dans la plupart des ménages enquêtés. Les consommations pour les différents usages domestiques révèlent que l'alimentation (cuisine et boisson) n'occupe que $5 \%$ de la consommation en eau potable, et la plus grande partie $(95 \%)$ est utilisée pour l'hygiène et le nettoyage (Tableau 2 ) où l'on trouve les deux usages domestiques les plus consommateurs en eau que sont les usages "WC" et "douche" qui représentent $86 \%$ de la consommation totale dont $43 \%$ pour chaque usage.

\section{Chasse d'eau et gaspillage}

Les ménages équipés de WC à chasse d'eau consomment en moyenne 112 litres par jour et par personne (Tableau 3), le WC seul consommant 53 litres d'eau (soit 46,4\%). Par contre les ménages équipés de WC sans chasse d'eau consomment en moyenne 83 litres par jour et par personne (Tableau 3), le WC seul consommant 31 litres soit 38,4\% (Tableau 4). On remarque que les WC à chasses d'eau ordinaires consomment 22 litres de plus par jour et par personne par rapport à la consommation lorsqu'ils ne sont pas équipés de chasses d'eau. Les chasses d'eau utilisées dans les ménages ne sont donc pas économiques, elles sont à la base de gaspillage d'eau.

\section{Test des quantités d'eau rejetées par les différents modèles de chasse d'eau dans les ménages sélectionnés.}

Les chasses d'eau ordinaires rencontrées dans les ménages sont à bouton poussoir ou à tirette avec l'unique possibilité d'exercer une seule action sur la chasse. Les quantités d'eau libérées après chaque action sur les différents modèles de chasse d'eau rencontrés dans les ménages montrent que ces chasses libèrent une quantité d'eau unique quel que soit le besoin (urine, selles, crachat, etc.) et presque identique au volume d'eau stocké dans le réservoir (Tableau 5). 
Cette quantité d'eau libérée après chaque action en poussant le bouton ou en tirant la tirette selon le modèle de chasse, ne dépend ni du mécanisme de la chasse, ni de la forme du réservoir du WC, mais plutôt du volume d'eau stocké dans le réservoir.

À contrario, les chasses d'eau économiques sont à bouton poussoir avec la possibilité d'exercer deux actions sur la chasse. Une première action consistant à appuyer sur le bouton et le relâcher aussitôt (après 1 à 2 secondes environ). Cette première permet l'évacuation des besoins autres que les selles et les vomissements. La seconde action permet d'évacuer les selles et vomissement, elle consiste à appuyer sur le bouton et le maintenir jusqu'à l'évacuation (3 à 5 secondes environ). Les volumes d'eau libérés par le modèle de chasse d'eau économique installé dans les cinq ménages montrent que les quantités d'eau libérées après chaque action ne sont pas identiques (Tableau 5). On remarque que la chasse d'eau économique ne libère pas toute l'eau $\mathrm{du}$ réservoir, pour l'ensemble des six mécanismes installés, les quantités d'eau libérées pour la selle correspondent au double de celles libérées pour évacuer l'urine. Aussi, les quantités d'eau libérées après chaque action sur la chasse d'eau économique dépendent non seulement du mécanisme de la chasse à l'intérieur du réservoir mais aussi de la forme du réservoir et du volume d'eau qui y est stocké.

\section{Suivi et comparaison des consommations en eau des modèles de chasses d'eau rencontrée dans chacun des ménages avec le modèle économique installé et économies d'eau réalisées}

Le suivi de la consommation en eau potable dans les 5 ménages sur une période totale de 42 jours (21 jours pour chaque modèle) a montré l'impact des chasses économiques sur la consommation en eau. En effet, pour les mêmes habitudes d'usage de l'eau, l'utilisation des chasses d'eau économiques a permis de réduire les consommations spécifiques par personne dans chacun des ménages aussi bien les jours ouvrables que les week-ends où les consommations maximales ont été le plus souvent observées. Le Tableau 6 montre les économies d'eau réalisées avec les chasses d'eau économique dans les cinq ménages. Le calcul des économies réalisées pour l'ensemble des 5 ménages a été fait à partir des moyennes journalières par personne obtenue dans chacune des phases. Les économies d'eau journalières réalisées se situent autour de 11 litres par personne.

Économie d'eau réalisable dans la ville d'Abidjan et réduction du déficit par l'utilisation des chasses d'eau économiques

La réalisation des économies d'eau au niveau des ménages passe par la réduction de la consommation d'eau au niveau des WC équipés de chasses d'eau qui sont les plus gros consommateurs domestiques d'eau potable. En effet, l'étude a montré que les chasses d'eau économiques donnent une possibilité de réduction d'eau allant de 11 à 27 litres (Tableau 7) faisant chuter la consommation de 112 litres à 101 litres au moins et au plus 85 litres par jour et par personne. Ces réductions représentent une économie de $20 \%$ à $51 \%$ sur la consommation moyenne du ménage. On réalise de même une économie de l'ordre de 11 à 13 litres (Tableau 7) sur la consommation pour les ménages équipés de chasse d'eau économique dont les WC n'étaient pas munis de chasse d'eau, soit une baisse de la consommation passant de 83 à 72 litres au moins et au plus 70 litres par jour et par personne. Cela correspond à une économie de 35 à $42 \%$.

Ainsi, si l'on estime que $51 \%$ des ménages à Abidjan (483 667 ménages) sont équipés de chasses d'eau pour une moyenne de 4,6 personnes par ménage (INS, 2014), une économie d'eau pour un ensemble de 2 224868 habitants entre 24000 à $60000 \mathrm{~m}^{3}$ d'eau/jour. De plus, sur les 100 ménages interrogés, 37 n'étaient pas équipés de WC à chasse d'eau. Si ces ménages (350 896 ménages) s'équipaient de chasses d'eau 
économique, ils réaliseraient une économie de 11 à 13 litres d'eau par jour et par personne. Cette part de la population qui correspond à 1614000 habitants pourrait réaliser alors une économie globale allant de $17000 \mathrm{~m}^{3}$ à $21000 \mathrm{~m}^{3}$ d'eau par jour. L'ensemble de la ville d'Abidjan pourrait, dans ce cas, réaliser rien que par l'utilisation des chasses d'eau économiques, une économie allant de $41000 \mathrm{~m}^{3}$ à $81000 \mathrm{~m}^{3}$ d'eau par jour soit un gain total de 14965000 à $29565000 \mathrm{~m}^{3}$ par an. Cette quantité d'eau économisée en termes de consommation spécifique et avec la même capacité de production actuelle de la Société de Distribution d'Eau de Côte d'Ivoire (SODECI), permettrait de couvrir les besoins en eau potable de 422000 à 835000 personnes de plus à Abidjan.

Tableau 1: Consommation moyenne journalière d'eau potable en litre par personne selon le niveau de standing des ménages.

\begin{tabular}{lccc}
\hline & $\begin{array}{l}\text { Bas } \\
\text { standing }\end{array}$ & $\begin{array}{l}\text { Moyen } \\
\text { standing }\end{array}$ & $\begin{array}{l}\text { Haut } \\
\text { standing }\end{array}$ \\
\hline Nombre de ménages & 51 & 45 & 4 \\
Consommation moyenne journalière & 88 & 105 & 132 \\
Écart-type & 27 & 20 & 11 \\
\hline Consommation moyenne journalière & & $\mathbf{9 7}$ & \\
totale & & $\mathbf{2 7}$ & \\
\hline Écart-type & & \\
\hline
\end{tabular}

Tableau 2: Consommation moyenne journalière d'eau potable (litres/jour/pers.) par usage domestique.

\begin{tabular}{lcccc}
\hline Usages & $\begin{array}{l}\text { Consommation } \\
\text { moyenne }\end{array}$ & $\begin{array}{l}\text { Consommation } \\
\text { médiane }\end{array}$ & $\begin{array}{l}\text { Écart- } \\
\text { type }\end{array}$ & $\begin{array}{l}\text { Pourcentage } \\
(\%)\end{array}$ \\
\hline WC & 42 & 40 & 13 & 43 \\
Douche & 42 & 40 & 13 & 43 \\
Vaisselle & 4 & 3 & 4 & 5 \\
Lessive & 3 & 3 & 2 & 3 \\
Cuisine & 3 & 2 & 2 & 3 \\
Eau de boisson & 2 & 2 & 1 & 2 \\
Nettoyage & 1 & 1 & 1 & 1 \\
Véhicule & 0 & 0 & 0 & 0 \\
Jardin & 0 & 0 & 0 & 0 \\
\hline Consommation Totale & $\mathbf{9 7}$ & $\mathbf{9 0}$ & $\mathbf{2 7}$ & $\mathbf{1 0 0}$ \\
\hline
\end{tabular}

Tableau 3: Consommations moyenne journalière des ménages équipés de WC.

\begin{tabular}{lcc}
\hline & $\begin{array}{c}\text { Consommation moyenne } \\
\text { journalière par personne en } \\
\text { Litres/jour }\end{array}$ & Écart-type \\
\hline Ménages équipés de WC à chasse d'eau & 112 & 17 \\
\hline Ménages équipés de WC sans chasse d'eau & 83 & 24 \\
\hline
\end{tabular}


Tableau 4 : Consommation journalière des WC à chasse d'eau et WC sans chasse d'eau.

\begin{tabular}{lcccc}
\hline & Pour la selle & Pour l'urine & $\begin{array}{l}\text { Consommation des } \\
\text { WC sans chasse }\end{array}$ & $\begin{array}{l}\text { Consommation des } \\
\text { WC à chasse }\end{array}$ \\
\hline $\begin{array}{l}\text { Moyenne } \\
\text { Jour/pers. }\end{array}$ & 1 & 4 & 31 & 53 \\
\hline $\begin{array}{l}\text { Écart- } \\
\text { type }\end{array}$ & 0,45 & 1,23 & 12,5 & 11,4 \\
\hline
\end{tabular}

Tableau 5 : Quantités d'eau rejetées par l'utilisation des chasses d'eau rencontrées dans les ménages sélectionnés.

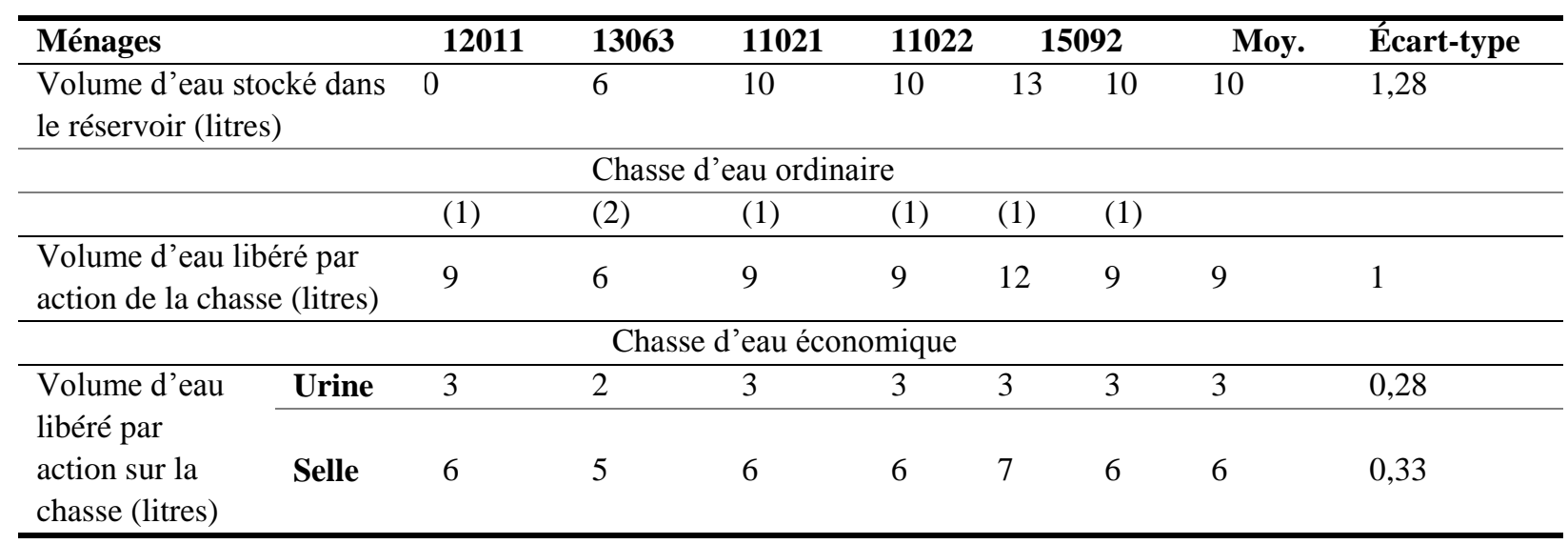
(1) Chasse d'eau à tirette
(2) Chasse d'eau à bouton poussoir

Tableau 6 : Tableau comparatif des consommations d'eau journalière globale des ménages sur modèles de chasses d'eau dans chacun des ménages sélectionnés.

\begin{tabular}{lccccc}
\hline Ménages & Commune & $\begin{array}{c}\text { Consommation } \\
\text { sur chasse } \\
\text { d'eau ordinaire }\end{array}$ & $\begin{array}{c}\text { Consommation } \\
\text { sur chasse d'eau } \\
\text { économique }\end{array}$ & $\begin{array}{c}\text { Economie } \\
\text { d'eau réalisée }\end{array}$ & $\begin{array}{c}\text { Pourcentage } \\
\text { d'économie }\end{array}$ \\
\hline 12011 & Adjamé & 75 & 64 & 11 & $14,7 \%$ \\
13063 & Yopougon & 87 & 76 & 11 & $12,6 \%$ \\
11021 & Cocody & 70 & 58 & 11 & $15,7 \%$ \\
11022 & Cocody & 145 & 133 & 12 & $08,3 \%$ \\
15092 & Marcory & 70 & 61 & 09 & $13 \%$ \\
\hline
\end{tabular}

Tableau 5: Economies d'eau réalisables sur les WC par l'utilisation des mécanismes économique dans les ménages équipés de WC.

\begin{tabular}{lccc}
\hline & $\begin{array}{c}\text { Consommation } \\
\text { initiale } \\
\text { (litre/jour/pers.) }\end{array}$ & $\begin{array}{c}\text { Économie réalisable } \\
\text { avec chasse d'eau } \\
\text { économique (litres) }\end{array}$ & Pourcentage d'économie \\
\hline WC à chasse d'eau & 53 & $11 \sim 27$ & $20 \sim 51 \%$ \\
WC sans chasse d'eau & 31 & $11 \sim 13$ & $35 \sim 42 \%$ \\
\hline
\end{tabular}




\section{DISCUSSION}

La moyenne de consommation journalière en eau par personne à Abidjan ( 97 litres) est largement au-dessus du minimum vital qui est de 20 litres d'eau par jour et par personne (OMS, 2015). Ces résultats sont confirmés par Sakou et al. (2012) qui ont montrés que les ménages disposant d'un accès permanent à l'eau potable consomment en moyenne 100 litres d'eau par jour et par personne. Cependant, cette valeur moyenne cache la disparité qui existe entre les différents niveaux de vie des populations. En effet, les consommations journalières d'eau potable des ménages varient selon le standing des différents ménages. Ces différences s'expliquent certes par le niveau de vie des ménages, mais aussi par l'inégalité de la fourniture d'eau entre les communes. Cette inégalité se traduit par les coupures d'eau fréquentes rencontrées dans les quartiers de bas standing, et qui selon l'enquête correspond à taux de $66 \%$ sur l'ensemble des coupures d'eau observé (Tableau 8).

Par ailleurs, la forte demande journalière d'eau potable à Abidjan, estimée à $427000 \mathrm{~m}^{3}$ selon l'étude, a engendré un déficit de $115000 \mathrm{~m}^{3}$. Face à ce déficit, l'État accrô̂t ses investissements pour mobiliser d'autres ressources en eau exploitables en vue de faire face à la forte demande. Ces efforts ont permis en 2014 la mobilisation de la nappe d'eau du sud Comoé situé à $59 \mathrm{~km}$ d'Abidjan dont le coût total d'investissement est de 90 milliards de francs CFA et pour une capacité de production journalière de $80000 \mathrm{~m}^{3} /$ jour d'eau sur une échéance de 15 ans avant de se tourner vers la mobilisation d'autres ressources en eaux. Ainsi, le déficit n'est réduit de $69,4 \%$ que pour un temps, il s'accentuera davantage dans le temps avec la croissance de la population. De ce fait, mobiliser uniquement sans cesse des ressources en eau de plus en plus loin n'est pas une solution durable.

Une alternative économique serait d'envisager une gestion rationnelle de la nappe d'Abidjan à travers la réduction des consommations d'eau dans les ménages branchés au Réseau Public d'Eau Potable exploité par la SODECI.

Selon les résultats de cette étude, la réalisation des économies d'eau au niveau des ménages passe par la réduction de la consommation d'eau au niveau des WC équipés de chasses d'eau qui sont les plus gros consommateurs domestiques d'eau potable. Ces réductions représentent une économie de $20 \%$ à $51 \%$ sur la consommation moyenne du ménage. Ces résultats sont conformes à ceux trouvés par l'Office Nationale de l'Eau Potable (2015) qui, dans les administrations de l'État, a montré que l'utilisation des chasses d'eau économiques a permis de réaliser des économies d'eau de l'ordre de 38\% pour l'ensemble des sites étudiés.

Au niveau de la ville d'Abidjan, certes la taille de l'échantillon d'étude appelle à une extrapolation prudente des résultats. Néanmoins, ces premiers résultats ont le méritent de mettre en exergue la possibilité d'économie d'eau à grande échelle au niveau des ménages à travers l'utilisation des chasses d'eau adéquates.

Par ailleurs, ces résultats suggèrent de conduire des études plus approfondies pour en tirer toutes les leçons. En attendant, des leçons partielles peuvent être tirées à partir des résultats actuels.

Ces résultats laissent donc penser qu'il peut avoir une alternative consistant à mieux gérer l'existant qui est tout près que d'aller chercher la ressource en eau toujours plus loin. En effet, équiper les ménages d'Abidjan en chasse d'eau économique revient à économiser $81000 \mathrm{~m}^{3}$ /jour pour un investissement estimé à 24 milliards de francs CFA (Tableau 9).

Or la mobilisation la nappe de Bonoua pour renforcer la capacité de production d'Abidjan avec un débit de $\left(80000 \mathrm{~m}^{3}\right.$ /jour $)$ a couté 90 milliards de francs CFA en investissement. Il faudrait ajouter à ce coût d'investissement les coûts de pompage qui sont absent dans le cas de l'économie d'eau par recours aux chasses d'eau économique. 
Tableau 8: Nombre de plainte lié aux coupures d'eau rencontré dans les ménages selon le niveau de standing.

\begin{tabular}{llccc}
\hline & & \multicolumn{2}{c}{ Coupures fréquentes } & \\
\cline { 3 - 4 } & & \multicolumn{2}{c}{ d'eau ? } & Total \\
\cline { 3 - 4 } Niveau de & Oui & Non & \\
standing & Moyen standing & 14 & 6 & 35 \\
& Haut standing & 1 & 29 & 43 \\
\hline Total & & 44 & 38 & 4 \\
\hline
\end{tabular}

Tableau 9 : Estimation du coût de d'équipement des ménages en chasse d'eau économique.

\begin{tabular}{lll}
\hline Désignation & \multicolumn{1}{c}{ Quantité } & Prix unitaire en FCFA \\
\hline Ménages équipés de WC partiel (cuvette sans réservoir) & \\
Chasse économique & 483667 & 15000 \\
Installation & 483667 & 5000 \\
\hline Coût partiel 1 en FCFA & 9673340000 \\
\hline Ménages équipés de WC complet (cuvette + réservoir) & \\
Chasse économique & 350896 & 15000 \\
Réservoir & 350896 & 15000 \\
Installation & 350896 & 10000 \\
\hline Coût partiel 2 en FCFA & 14035840000 \\
\hline Coût total d'investissement (FCFA) & $\mathbf{2 3 7 0 9 ~ 1 8 0 ~ 0 0 0}$ \\
\hline
\end{tabular}

\section{Conclusion}

L'objectif de cette étude a été d'évaluer l'économie d'eau potable réalisable dans la ville d'Abidjan par l'utilisation des chasses d'eau sanitaires économique dans les ménages. Il en ressort que la consommation moyenne journalière des ménages en eau potable dans la ville d'Abidjan est de 97 litres par personne. Les deux grands besoins domestiques consommateurs d'eau dans les ménages sont les usages WC et douche, qui représentent $86 \%$ de la consommation totale en eau potable. L'utilisation des chasses d'eau économique permet de réaliser une économie journalière allant de 11 à 27 litres d'eau par personne. Cette économie correspond pour l'ensemble de la ville d'Abidjan à un gain journalier de $41000 \mathrm{~m}^{3}$ à $81000 \mathrm{~m}^{3} \mathrm{~d}$ 'eau. Il serait intéressant en perspective de confirmer l'économie de l'eau à travers les chasses économique par une étude plus approfondie et plus étendue à d'autres modèles de chasse d'eau, d'étudier le degré d'acceptabilité sociale de ces nouveaux produits et mieux d'explorer les modèles d'affaires qui pourraient conduire à la mise en œuvre d'une telle innovation. Toute chose qui une fois possible, s'inscrirait sans conteste dans la lignée des "solutions vertes » permettant de faire face aux changements climatiques dont les effets ne 
sont que déplorables dans le secteur de l'eau potable.

\section{CONFLIT D'INTÉRÊTS}

Les auteurs déclarent qu'il n'y a aucun conflit d'intérêts.

\section{CONTRIBUTIONS DES UTEURS}

TBC a collecté les données de terrain, rédigé le manuscrit, conçu les cartes et figures. KS a fournis les chasses d'eau économique. SK, GT et SG ont contribué à la lecture, à l'amélioration et à la validation du manuscrit.

\section{REMERCIEMENS}

Les auteurs remercient l'Association Africaine de l'Eau (AAE) pour le matériel et tous les moyens mis à leur disposition pour réaliser cette étude.

\section{RÉFÉRENCS}

Bacharou T, Houinou G, Adjovi C, Adjiboicha M. 2012. Régime de consommation en eau et son utilisation dans le calcul des réseaux d'alimentation en eau potable. Rev. Ivoir. Sci. Technol., 19: 159-174. DOI :

http://revist.net/REVIST_19/REVIST_1 9_11.pdf

Baidai Y. 2011. Analyse de cycle de vie appliquée à un système de production d'eau potable : cas de l'unité industrielle SODECI nord-riviera. Institut de Formation à la Haute Expertise et de Recherche - Master II Génie de l'Environnement. $38 \mathrm{p}$. https://www.memoireonline.com/10/13/ 7512/Analyse-de-cycle-de-vieappliquee--un-systeme-de-productiond-eau-potable--cas-de-l-unite-indu.html Bureau National d'Etude Technique et de Développement (BNETD). 2015. Projet de renforcement de la voirie d'Abidjan cas des itinéraires des communes d'Abobo, plateau et Treichville. Étude d'impact environnemental et social. 223p. http://documents.worldbank.org/curated /en/568941468172746492/text/E3029V7-FRENCH-P124715-EIES-

Renforcement-dAbidjan-Box391438BPUBLIC.txt.

Centre de Promotion des Investissements en Côte d'Ivoire. 2005. Présentation de la loi n $98-755$ du 23 Décembre 1998 portant Code de l'Eau. Version $\mathrm{N}^{\circ} 1$ AD-DAD-CEPICI. 11p.

De Viguerie P. 2009. Les usages domestiques de l'eau. 162p. http://www.ladocumentationfrancaise.fr /rapportspublics/094000238/index.shtml

Durand C. 2002. Méthodes de sondage SOL3017. Département de sociologie. Université de Montréal. 77p.

Ettien B. 2010. Etude de faisabilité de l'utilisation de la lagune aghien pour l'alimentation en eau potable du district d'Abidjan. Génie sanitaire et environnement. Institut Nationale d'ingénierie de d'eau et environnement (2ie). $70 \mathrm{p}$.

Institut Nationale De La Statistique (INS). 2014. Recensement Général de la Population et de l'Habitat 2014. Résultats globaux. 22p.

Koffi Y, Ahoussi K, Kouassi A, Kpangui L, Biemi J. 2014. Modélisation de la consommation en eau potable dans les capitales africaines au sud du Sahara : application des Réseaux de neurones formels à la ville de Yamoussoukro, capitale politique de la Cote d'Ivoire, Journal of Asian Scientific Research, 2(10) : 562-573.

Marin P, Ouayoro E, Fall M, Verspyck R. 2009. Un partenariat réussi pour l'eau en Côte d'Ivoire Leçons tirées de 50 ans de gestion privée efficace. $4 \mathrm{p}$.

Mirad Blondie. 2004. La consommation et les usages domestiques de l'eau dans le pays-cœur-entre-deux-mers, Génie de l'environnement - Université Michel de Montaigne Bordeaux III. 69p. 
Nassartebaye N. 2011. Accès à l'eau potable et à l'assainissement ; quels enjeux pour la santé dans les quartiers précaires ? Etude appliquée au quartier Gamkallé de la commune IV de Niamey au Niger, Université Abdou Moumouni de Niamey. 59p.

Office Nationale de l'Eau Potable (ONEP). 2015. Rapport de la phase 1 du projet Économique. 34p.

Oga Y, Koffi K, Yao N, Kpan J, Kouamé Y, Biémi J. 2016. Caractéristiques hydrogéologiques de l'intervalle 177 $79 \mathrm{~m} \mathrm{du}$ forage d'Abouabou (Abidjan, Côte d'Ivoire) : paramètres physicochimiques. International Journal of Innovation and Scientific Research, 21(1): 2351-8014. DOI : https://www.researchgate.net/publicatio n/312098282_Caracteristiques_hydroge ologiques_de_l'intervalle_177_79_m_d u_forage_d'Abouabou_Abidjan_Cote_d' Ivoire_parametres_physico-chimiques

OMS. 2004. Directives de qualité pour l'eau de boisson (3ème édn, Vol. 1). OMS ; $110 \mathrm{p}$.

OMS. 2015. Rapport 2015 du JMP : faits essentiels. OMS ; 4p.

OMS/UNICEF. 2007. Atteindre l'OMD relatif à l'eau potable et à l'assainissement, le défi urbain et rural de la décennie. 48p.

OMS/UNICEF. 2008. Un Aperçu de la Situation de l'Eau Potable en Afrique, $11^{\text {ème }}$ Sommet des Chefs d'Etat et de Gouvernement de l'Union Africaine sur le thème : Réalisation des Objectifs du Millénaire concernant l'Eau et l'Assainissement. 13p.

OMS/UNICEF. 2012. Un Aperçu de la Situation de l'Eau Potable et de l'Assainissement en Afrique. 24p.

Plan National de Développement. 2016-2020 (PND2016-2020). 2014. Diagnostic stratégique de la Côte d'Ivoire sur la trajectoire de l'Emergence, Tome 1. $119 \mathrm{p}$.

http://www.caidp.ci/uploads/4b01aedeff 1d6c1e74fa1344f6e6cb10.pdf
Rayaleh H. 2004. La gestion d'une pénurie d'eau à Djibouti, Institut de recherche pour le développement. Thèse en géographie. Université d'Orléans. 315p. https://www.pseau.org/outils/ouvrages/i rd_gestion_penurie_eau_djibouti_these rayaleh.pdf

Romy H. 2008. Les nations unies et le problème de l'eau dans le monde : essai sur ONU-EAU, Université du Québec à Montréal. https://archipel.uqam.ca/1414/1/M1045 1.pdf

Sackou K, Oga S, Claon S, Bama M, Mbrah K, Houenou Y, Kouadio K. 2010. Conditions d'accès et de stockage de l'eau : enquête dans les ménages en zone périurbaine à Abidjan en 2010. Santé Publique 2/2012, 24 : 133-142 DOI : https://www.cairn.info/revuesante-publique-2012-2-page-133.htm

Silue B, Cisse G, Kone B, Zurbrugg C, Savane I. 2012. Equité d'accès à l'eau Potable dans un contexte de diversité de modes d'approvisionnement : cas de la ville de Bouaké (Côte D'ivoire), European Journal of Scientific Research 72(2): 298-310. DOI : https://www.pseau.org/outils/ouvrages/e jsr_equite_d_acces_a_1_eau_potable_da ns_un_contexte_de_diversite_de_mode s_d_approvisionnement_cas_de_la_vill e_de_bouake_cote_d_ivoire_2012.pdf

SODECI. 2010. Rapport de gestion 2010. 21p. http://www.sodeci.ci/rapport-degestion

SODECI. 2014. Rapport de gestion 2014. 32p. http://www.sodeci.ci/rapport-degestion

Soglo Y, Nlombi K, Et Taladidia T. 2002. Détermination de la demande d'eau potable par la création d'un marché fictif base sur le consentement à payer : cas de la Ville de Cotonou au Benin. 22p.

Soro N, Ouattara L, Dongo K, Kouadio K, Ahoussi K, Soro G, Oga M, Savane I, Biemi J. 2010. Déchets municipaux dans le district d'Abidjan en Côte 
d'Ivoire : sources potentielles de pollution des eaux souterraines, Int. J. Biol. Chem. Sci., 4(6): 2203-2219. https://www.researchgate.net/publicatio n/312115955_Dechets_municipaux_dan s_le_District_d'Abidjan_en_Cote_d'Ivoi re_source_potentielles_de_pollution_de s_eaux_souterraines

Sy I, Keita M, Traore D, Kone B, Ba K, Boilil O, Fayomi B, Bonfoh B, Tanner M, Cisse G. 2014. Eau, hygiène, assainissement et santé dans les quartiers précaires à Nouakchott (Mauritanie) : contribution à l'approche écosanté à Hay Saken. 25p.

Tia L. 2015. Acteurs privés et approvisionnement en eau potable des populations de la commune d'AboboCôte d'Ivoire. Revue Canadienne de Géographie Tropicale/Canadian Journal of Tropical Geography, 2(2). Mis en ligne le 15 novembre 2015. 1528. http://laurentienne.ca/rcgt
Tuo P. 2010. Assainissement et gestion de l'environnement dans la commune d'Adjamé : le cas de Williamsville (Abidjan-Cote d'Ivoire), Institut de Géographie Tropicale-Université Nationale d'Abidjan-Cocody .Doctorant en Géographie Option Environnement et Santé 2010. 13p.

UNICEF. 2013. Progrès en matière d'assainissement et d'alimentation en eau, rapport 2013. 40p.

UNICEF/BURKINA FASO. 2009. L'eau potable, les toilettes et l'hygiène pour tous. 40p.

Yacine B, Gaudremeau J, Gerbe B, Khamsing F, Rabatel Y. 2004. Eau ressources et menaces. 30p.

Yélognissè Coffi L. 2007. L'amélioration des conditions d'accès à une eau potable pour l'eau de boisson dans les milieux ruraux du bénin : étude des pratiques locales.Université du Québec à Montréal. 117p. 